\title{
Expression of versican mRNA transcript to predict cardiac remodeling after myocardial infarction
}

\author{
Aleksandra Chabior*, Aleksandra Gąsecka1*, Michał Marchel', Roksana Gozdowska', Agnieszka Makowska', \\ Karolina Maciak², Monika Góra², Krzysztof J Filipiak', Beata Burzyńska², Grzegorz Opolski \\ ${ }_{11}^{1 \text { st }}$ Chair and Department of Cardiology, Medical University of Warsaw, Warszawa, Poland \\ Institute of Biochemistry and Biophysics, Polish Academy of Sciences, Warszawa, Poland \\ *Both authors equally contributed to the study and share the first authorship..
}

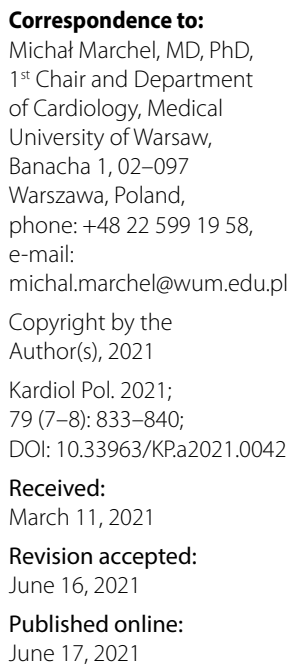

\section{A B S T R A C T}

Background: Adverse left-ventricular remodeling (LVR) is defined as a $20 \%$ increase in end-diastolic left-ventricular volume 6 months after acute myocardial infarction (AMI). LVR is associated with cardiac dysfunction, therefore deteriorating the prognosis.

Aims: We aimed at comparing the concentrations of messenger RNA transcripts in the peripheral blood of patients with and without LVR at 6 months.

Methods: The study included 75 patients with first ST-elevation myocardial infarction (STEMI) treated with percutaneous coronary intervention. Whole blood concentrations of 6 transcripts were determined 24 hours after AMI using droplet digital polymerase chain reaction. The correlations between mRNA transcript expression and left ventricular ejection fraction (LVEF) and N-terminal-pro B type natriuretic peptide (NT-proBNP) concentration were evaluated.

Results: Among 75 patients, 4 were lost to follow-up, and 71 were included in the analysis. Seventeen (24\%) patients developed LVR at 6 months. Versican (VCAN) mRNA expression was lower in patients who developed LVR, compared to those who did not $(P=0.02)$, and discriminated between these patients (area under the ROC curve $67 \% ; P=0.04$ ). Expression of VCAN transcript $<75.3$ normalized units predicted LVR with $71 \%$ sensitivity and $67 \%$ specificity. In a multivariable regression analysis, VCAN expression remained the only independent predictor of LVR (OR 3.475; 95\% Cl, 1.000-12.075; $P=0.04)$.

Conclusions: Dysregulation of VCAN expression in the acute phase of AMI may contribute to LVR at 6 months. Whether decreased expression of VCAN might be a useful tool to predict LVR in clinical practice remains to be established.

Key words: cardiac remodeling, mRNA transcripts, myocardial infarction, versican

Kardiol Pol 2021; 79, 7-8: 833-840

\section{INTRODUCTION}

Heart failure (HF) is an important public health challenge, that occurrs in $1 \%-2 \%$ of the adult population, and this prevalence increases to $10 \%$ among people over 70 years of age [1]. Acute myocardial infarction (AMI) is responsible for over $50 \%$ of cases of HF [2]. In patients with AMI, the loss of contractile tissue and left ventricular remodeling (LVR) anticipated by the structural and functional alterations to the left ventricle, including fibrosis and inflammation [3]. The most common definition of LVR, applied also in the present study, is a $20 \%$ increase in left ventricular end-diastolic volume (LVEDV) at 6 months after AMI [4]. LVR affects 25\% of patients after AMI and deteriorates the prognosis [5]. Clinical, biochemical, and imaging parameters increasing the risk of $L V R$ after $A M I$ include the extension and anterior location of AMI, late or unsuccessful revascularization [6], high concentrations of natriuretic peptides and cardiac troponins [7], and enlarged left ventricle (LV) on echocardiography [8]. However, at present, there are no reliable biomarkers to predict cardiac remodeling. Due to their role in the development of adverse cardiac remodeling after AMI, novel markers including microRNA and messenger RNA (mRNA) transcripts might be superior to the currently available biomarkers of HF and cardiac remodeling, such as N-terminal pro-B-type natriuretic peptide (NT-proBNP). Hence, identification of the crucial genes involved in the process of remodeling and associated mRNA transcripts might help to individualize the treatment strategy and 
WHAT'S NEW?

Cardiac remodeling after acute myocardial infarction is associated with ventricular dysfunction and deteriorates the prognosis. Remodeling involves genetic and molecular changes in response to cardiac injury. The present study tested the concentrations of mRNA transcripts in the peripheral blood of patients after myocardial infarction with and without cardiac remodeling. Our results revealed that versican (VCAN) expression was lower in patients who developed left ventricular remodeling (LVR), compared to those who did not. Further studies are needed to assess whether VCAN might be a useful tool to predict cardiac remodeling in clinical practice.

improve cardiovascular risk stratification and prediction of outcomes [9].

Messenger RNA is a single-stranded molecule of ribonucleic acid that corresponds to the sequence of a gene and is read in the process of translation (synthesizing a protein) [10]. Previously, we showed that the expression of mRNA transcripts is altered in the peripheral blood of patients after AMI, compared to controls [11]. Using genome-wide gene expression profiling, we selected several transcripts involved in the development of HF after AMI. Here, we hypothesized that the post-AMI expression of mRNA differs in patients with and without LVR. We compared the level of selected mRNA transcripts in patients after AMI with and without LVR and evaluated their predictive value for LVR.

\section{METHODS}

\section{Study design}

We designed an investigator-initiated, prospective study, conducted at the $1^{\text {st }}$ Chair and Department of Cardiology, Medical University of Warsaw, Poland, in collaboration with the Institute of Biochemistry and Biophysics, Polish Academy of Sciences, Warsaw, Poland. The study protocol, designed in compliance with the Declaration of Helsinki, was approved by the Ethics Committee of the Medical University of Warsaw (KB/176/2015). All participants provided written informed consent. The study design is showed in Figure $1 \mathrm{~A}$.

\section{Study participants}

Participant inclusion and exclusion criteria are listed in Table 1. Patients were eligible for enrolment if they (a) were admitted to the hospital due to ST-segment elevation of acute myocardial infarction (STEMI), and (b) underwent percutaneous coronary intervention $(\mathrm{PCl})$ with stent implantation. STEMI was diagnosed based on persistent ST-segment elevation of at least $0.1 \mathrm{mV}$ in at least two contiguous electrocardiography leads or a new left bundle-branch block [12].

\section{Clinical data collection}

At baseline, data regarding demographics (age, gender), body mass index, cardiovascular risk factors, routine laboratory parameters. All patients received standard treatment after STEMI according to the guidelines, including dual antiplatelet therapy, $\beta$-blocker, angiotensin-converting

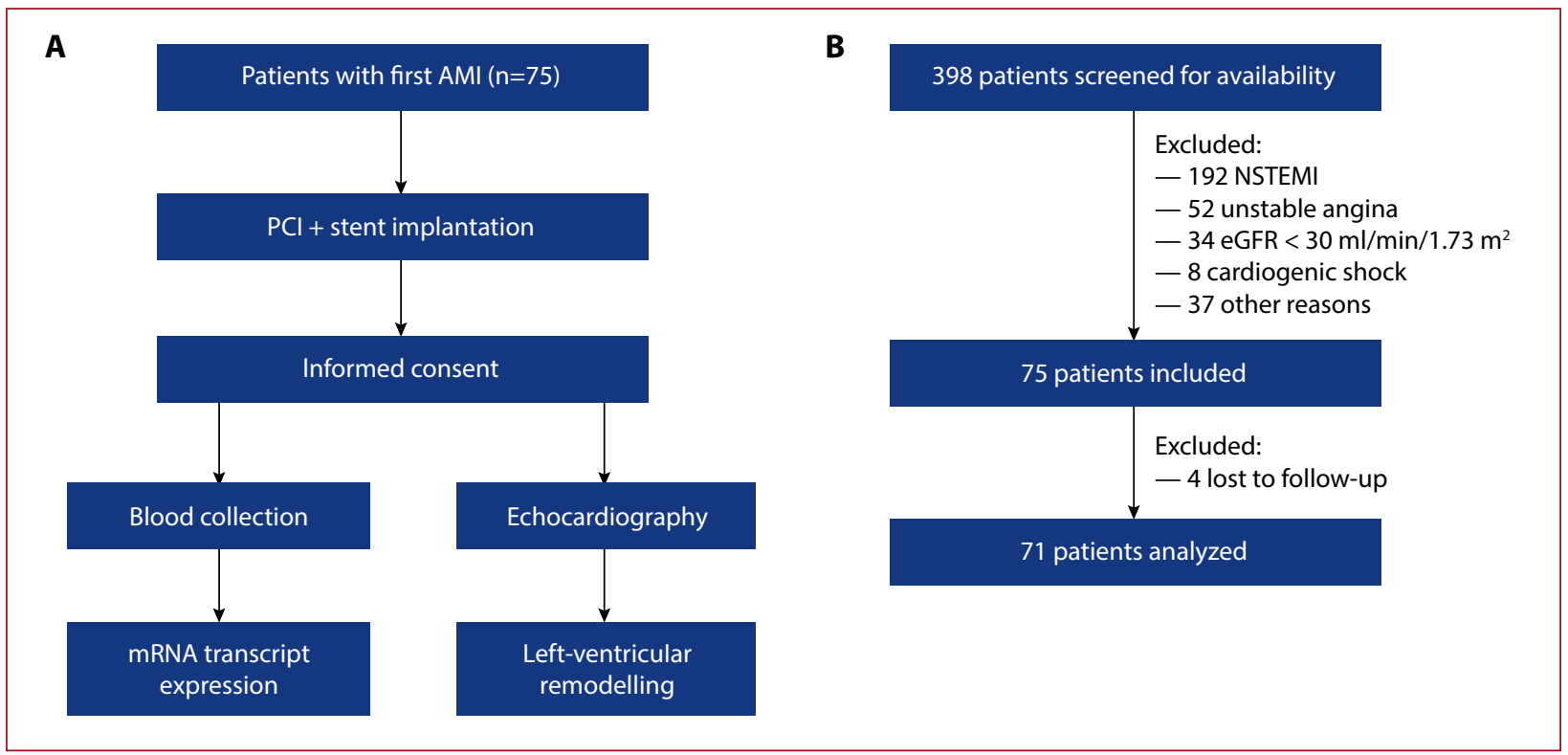

Figure 1. Study design and flow chart

Abbreviations: AMI, acute myocardial infarction; eGFR, estimated glomerular filtration rate; mRNA, messenger RNA; NSTEMI, non-ST-elevation myocardial infarction; $\mathrm{PCl}$, percutaneous coronary intervention 
Table 1. Eligibility criteria for the study

\begin{tabular}{|c|c|}
\hline Inclusion criteria & Exclusion criteria \\
\hline $\begin{array}{l}\text { Age } \geq 18 \text { years } \\
\text { Informed consent to participate in } \\
\text { the study } \\
\text { ST-elevation myocardial infarction } \\
\text { PCI with stent implantation }\end{array}$ & $\begin{array}{l}\text { Non-ST-elevation acute coronary } \\
\text { syndrome } \\
\text { Cardiogenic shock } \\
\text { Severe chronic renal failure } \\
\text { (eGFR }<30 \mathrm{ml} / \mathrm{min} / 1.73 \mathrm{~m}^{2} \text { ) } \\
\text { Severe liver insufficiency } \\
\text { (Child-Pugh class C) } \\
\text { Known pregnancy or breast-fe- } \\
\text { eding }\end{array}$ \\
\hline
\end{tabular}

Abbreviations: eGFR, estimated glomerular filtration rate; $\mathrm{PCl}$, percutaneous coronary intervention

enzyme inhibitor or angiotensin receptor blocker, aldosterone receptor antagonist, and protein pump inhibitor [12].

The concentration of NT-proBNP was measured within 24 hours after $\mathrm{AMI}$ and at 6-month follow-up in whole blood sample. Transthoracic echocardiography was conducted within 24 hours after AMI and repeated at the follow-up visit. LVR was defined as a $20 \%$ increase in LVEDV at6 months after AMI, compared to baseline echocardiography.

\section{Messenger RNA transcripts analysis}

Whole blood concentrations of 6 transcripts (ribonuclease 1 [RNASE1], Jun dimerization protein 2 [JDP2], cluster of differentiation 163 [CD163], cytochrome P450 family 27 subfamily A member 1 [CYP27A], versican [VCAN], interleukin type 1, receptor 2 [IL1R2]) were determined within 24 hours after AMI using droplet digital polymerase chain reaction (ddPCR). The selection of specific transcripts was based on the results of our earlier study, which showed that the genes responsible for the expression of these transcripts differ between patients with AMI and healthy controls, and between patients who developed post-infarct HF at 6 months vs those who did not [11]. In the past study, the expression of genes encoding the selected transcripts differed two-fold to three-fold between the groups (post-infarct HF vs no HF: RNASE1 2.044-fold change; $P=0.002 ;$ JDP2 1.825-fold change; $P=0.001 ; C D 163$ : 1.768fold change, $P=0.003$; IL 1R2: 3.167-fold change; $P=0.008$; AMI vs controls: $C Y P 27 A$ 1.712-fold change; $P=0.005$; VCAN 1.16-fold change; $P=0.001$ ). In the past study, we used both peripheral blood mononuclear cells and whole blood as the RNA source. The obtained results showed that the choice of material (whole blood vs peripheral blood mononuclear cells) and analytical method (microarrays vs RT-qPCR) does not affect the obtained results, indicating the robustness of the proposed approach. In the current research, using a different cohort of patients and different methodology (ddPCR), we focused on the evaluation of previously selected $m$ RNA transcripts in patients with and without LVR. Briefly, whole blood was collected into PAXgene Blood RNA tubes and total RNA was isolated using the PAXgene Blood RNA kit (Qiagen, Hilden, Germany). Reverse transcription was performed using $400 \mathrm{ng}$ of total RNA and the QuantiTect Reverse Transcription kit
(Qiagen). Primer sets were designed using Clone Manager Professional Suite software, version 6.00 (Sci Ed Software, NC, USA). Droplet digital PCR was carried out using QX200 ${ }^{\mathrm{TM}}$ Droplet Digital ${ }^{\mathrm{TM}}$ PCR System (Bio-Rad, CA, USA). The cDNA templates were diluted to a concentration of $4 \mathrm{ng} / \mu \mathrm{l}$ (total RNA equivalent) with nuclease-free water and $2 \mu$ l was assayed in a $22 \mu \mathrm{l}$ reaction volume using EvaGreen (BioRad) and thermal cycling conditions recommended for EvaGreen assays. After amplification, the plates were loaded into the QX200 Droplet Reader and the ddPCR results were analyzed with QuantaSoft software v.1.7.4.0917 and QuantaSoft Analysis Pro software v.1.0.596 (Bio-Rad). The absolute number of target molecules in the ddPCR reaction was determined using Poisson statistical analysis and background-corrected based on the no-template control. Data were expressed as normalized units, according to mRNA level of the reference gene HPRT1. In our study, we applied the droplet digital PCR approach to determine the mRNA expression level of studied genes. Droplet digital PCR fractionates templates into individual reaction systems, then transitional PCRs are run in individual wells and DNA content can be quantitated by direct counting PCR positive percentage among all reactions. The use of absence and presence of signals to indicate target DNA makes a'digital'direct measurement of samples. The digital PCR concept has many potential advantages over classical real-time $P C R$, including the capability to obtain absolute quantification without external references and robustness to variations.

\section{Endpoints}

The primary endpoint was the difference between the expression of mRNA transcripts after AMI in patients with and without LVR at 6 months. The secondary endpoint was the predictive value of mRNA transcripts for LVR at 6 months.

\section{Statistical analysis}

About $25 \%$ of patients after AMI develop LVR [5]. Since there are currently no data in the literature regarding the differences in mRNA transcript expression of the investigated genes in patients with and without LVR, our sample size calculation was based on the previous study, where we investigated gene expression in patients who did and did not develop post-infarct HR 6 months after STEMI [11]. In this study, the expression of most genes differed two-fold to three-fold between the groups, resulting in the nominal difference of at least 1 . Hence, the sample size was calculated based on the following assumptions: (I) significance level for two-sided testing 0.05; (II) test power 0.8; (III) standard deviation \pm 1 ; (IV) estimated difference in mean transcript expression between the group with and without LVR 1. Based on these assumptions, the study should include at least 17 patients who will develop LVR (25\% of the group). Assuming $10 \%$ of patients lost to follow-up, the required sample size was 75 patients. 
Table 2. Main characteristics of patients with and without left ventricular remodeling at 6 months after ST-elevation myocardial infarction

\begin{tabular}{|c|c|c|c|}
\hline Characteristic & LVR $(n=17)$ & No LVR $(n=54)$ & $P$-value \\
\hline Age, years, mean (SD) & $56.0(6.8)$ & $57.9(11.4)$ & 0.039 \\
\hline Male gender, $\mathrm{n}(\%)$ & $10(59)$ & $40(74)$ & 0.229 \\
\hline $\mathrm{BMI}, \mathrm{kg} / \mathrm{m}^{2}$, mean (SD) & $28.9(4.3)$ & $27.3(4.3)$ & 0.293 \\
\hline Anterior AMI, n (\%) & $6(35)$ & $15(36)$ & 0.135 \\
\hline \multicolumn{4}{|l|}{ Cardiovascular risk factors, $\mathrm{n}(\%)$} \\
\hline Arterial hypertension & $8(47)$ & $29(69)$ & 0.413 \\
\hline Diabetes mellitus & $3(18)$ & $15(36)$ & 0.845 \\
\hline Dyslipidemia & $10(59)$ & $30(71)$ & 0.075 \\
\hline Smoking & $6(35)$ & $26(62)$ & 0.908 \\
\hline Previous AMI & 1 (6) & $2(5)$ & 0.486 \\
\hline \multicolumn{4}{|l|}{ Laboratory parameters at baseline } \\
\hline NT-proBNP, pg/ml, median (IQR) & $1055(473-2076)$ & $607(269-1872)$ & 0.154 \\
\hline Creatinine, mg/dl, mean (SD) & $0.9(0.2)$ & $0.8(0.2)$ & 0.578 \\
\hline Hemoglobin, g/dl, mean (SD) & $14.2(0.8)$ & $14.3(2.1)$ & 0.847 \\
\hline Troponin I, ng/ml, median (IQR) & $25.8(6.2-41.9)$ & $14.6(3.6-54.2)$ & 0.630 \\
\hline \multicolumn{4}{|l|}{ Laboratory parameters at 6 months } \\
\hline NT-proBNP, pg/ml, median (IQR) & $96(82-356)$ & $228(113-347)$ & 0.207 \\
\hline \multicolumn{4}{|l|}{ Echocardiography at baseline, mean (SD) } \\
\hline LVEDV, ml & $102.8(27.5)$ & $118.6(45.6)$ & 0.343 \\
\hline LVESV, $\mathrm{ml}$ & $53.5(18.7)$ & $64.7(29.7)$ & 0.312 \\
\hline LVEF, \% & $48.9(7.8)$ & $46.2(8.9)$ & 0.823 \\
\hline \multicolumn{4}{|l|}{ Echocardiography at 6 months, mean (SD) } \\
\hline LVEDV, ml & $152.7(46.9)$ & $115.5(31.7)$ & 0.043 \\
\hline LVESV, $\mathrm{ml}$ & $65.3(28.1)$ & $56.3(25.3)$ & 0.350 \\
\hline LVEF, \% & $57.8(8.5)$ & $52.5(8.8)$ & 0.393 \\
\hline \multicolumn{4}{|l|}{ Pharmacotherapy at discharge, $\mathrm{n}(\%)$} \\
\hline Aspirin & $17(100)$ & $54(100)$ & 1.00 \\
\hline P2Y12 inhibitor & $17(100)$ & $54(100)$ & 1.00 \\
\hline Statin & $17(100)$ & $53(98)$ & 0.57 \\
\hline$\beta$-blocker & $15(88)$ & $52(96)$ & 0.21 \\
\hline ACE-inhibitor or ARB & $16(94)$ & $53(98)$ & 0.38 \\
\hline Aldosterone receptor antagonist & $2(1)$ & $5(10)$ & 0.76 \\
\hline
\end{tabular}

Abbreviations: $A C E$, angiotensin-converting enzyme; $A R B$, angiotensin-receptor blockers; BMI, body mass index — weight in kilograms divided by square of the height in meters; IQR, interquartile range; LVEDV, left ventriclar end-diastolic volume; LVEF, left ventriclar ejection fraction; LVESV, left ventriclar end-systolic volume; LVR, left ventricular remodeling; NT-proBNP, N-terminal pro-B-type natriuretic peptide; SD, standard deviation

Statistical analysis was conducted using IBM SPSS Statistics, version 27.0 (IBM). Categorical variables were presented as number and percent and compared using Fischer's exact test. A Shapiro-Wilk test was used to assess normal distribution of continuous variables. Continuous variables were presented as mean (SD) or median with interquartile range. Correlations were analyzed using a Spearman correlation coefficient test. Transcript expressions were compared between the groups using an unpaired t-test or Mann-Whitney $U$ test. The diagnostic ability of mRNA transcripts to discriminate between patients with and without LVR and the cut-offs were calculated using a receiver operating characteristic (ROC) curve. For analysis of LVR predictors, univariable logistic regression analysis was used. Next, multivariable analysis was performed, where LVR was used as a dependent variable and predictors which were significant in univariable analysis were used as independent variables. Results were expressed as odds ratio $(\mathrm{OR})$ and $95 \%$ confidence interval $(\mathrm{Cl})$. Mortality and other adverse events were reported descriptively. A $P$ value below 0.05 was considered significant.

\section{RESULTS}

The inclusion and exclusion charts are shown in Figure 1B. Between March 2015 and April 2019, 74 patients with first STEMI treated with $\mathrm{PCl}$ provided informed written consent and were included in the study.

\section{Clinical data}

Among 75 patients, 4 were lost to follow-up and 71 were included in the analysis. Patient characteristics are shown in Table 2. Seventeen (24\%) patients did and 54 patients (76\%) did not develop LVR at 6 months. Patients with LVR were younger than patients without LVR (mean age, 56.0 [SD, 6.8 ] years vs 57.9 [SD, 11.4] years, respectively; $P=0.039$ ). There were no other clinical differences between patients with and without LVR. Baseline echocardiography parameters and pharmacotherapy were comparable between the groups. All patients received dual antiplatelet therapy, all patients except for one received statin, and more than $88 \%$ of patients received a $\beta$-blocker and an angiotensin-converting enzyme inhibitor. 


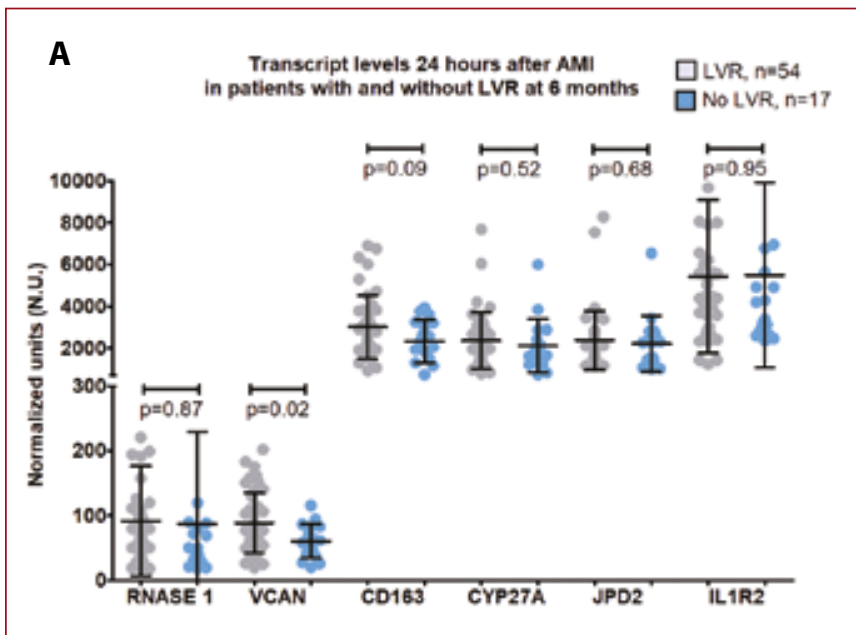

B
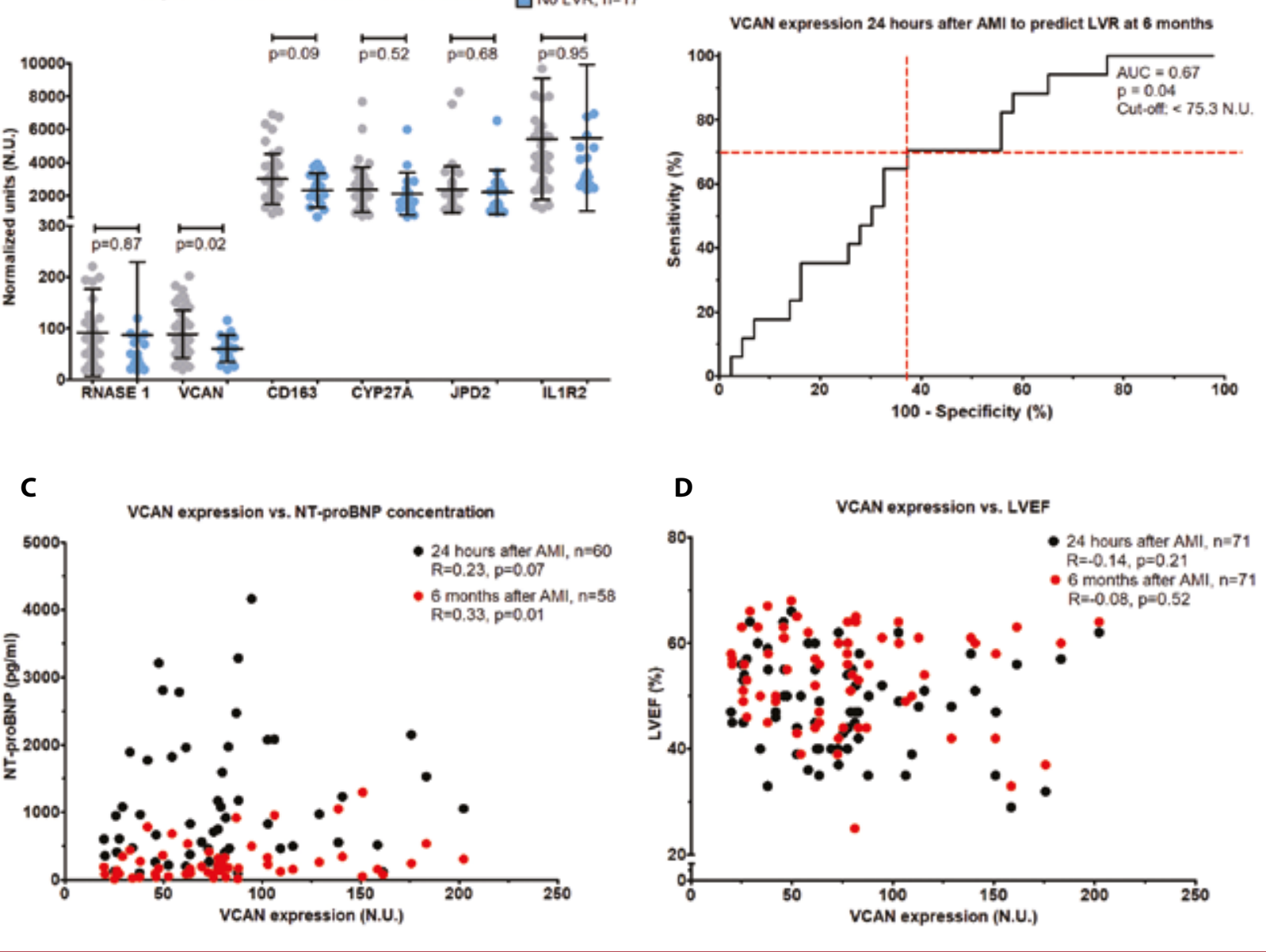

Figure 2. A. Messenger RNA transcript expression levels measured at baseline after AMI in patients with or without LVR at 6 months follow-up. Data are expressed as median and interquartile range and compared using Mann-Whitney U-test. B. Receiver operating characteristic curve showing VCAN expression after AMI to predict LVR. C. Correlation between VCAN expression measured at baseline and NTproBNP concentration measured at baseline and 6 months after AMI, analyzed using Spearman correlation coefficient test. D. Correlation between VCAN expression measured at baseline and LVEF at baseline and 6 months after AMI, analyzed using Spearman correlation coefficient test

Abbreviations: LVEF, left ventricular ejection fraction; LVR, left ventricle remodeling; VCAN, versican; other see Figure 1

At 6 months follow-up LVEDV was larger with patients with LVR, compared to those without LVR $(P=0.04)$. The LVEF was comparable in both groups. There were no deaths and only one recurrent hospitalization due to HF during the study in a patient from the LVR group.

\section{VCAN expression is an independent predictor of LVR}

Figure 2 shows the mRNA transcripts expression after AMI in patients with and without LVR at 6 months. VCAN expression was lower in patients who developed LVR, compared to those who did not $(P=0.02$; Figure $2 \mathrm{~A})$, and discriminated between these patients (area under the ROC curve, 67\%; $P=0.04$; Figure $2 \mathrm{~B}$ ). The expression of other mRNA transcripts did not differ between the groups. Expression of VCAN transcript $<75.3$ normalized units predicted LVR with $71 \%$ sensitivity and $67 \%$ specificity. Statistical estimates for the prediction of LVR by VCAN expression, including the cut-off values determined based on the ROC curve, are shown in Table 3.

To determine the clinical predictors of LVR, we performed an univariable logistic regression analysis (Table 4). We found that VCAN expression level below the determined cut-off and age were the only predictors of LVR (OR, 4.050; 95\% Cl, 1.204-13.619; $P=0.024$ for VCAN; OR, $0.944 ; 95 \%$ $\mathrm{Cl}, 0.892-0.999 ; P=0.047$ for age). Next, we incorporated VCAN expression and age in the multivariable regression model. VCAN was the only independent predictor of LVR (OR, 3.475; 95\% Cl, 1.000-12.075; $P=0.047$; Table 5).

\section{VCAN expression correlates with NT-proBNP concentration}

Figures $2 \mathrm{C}$ and $2 \mathrm{D}$ show the correlations between VCAN expression at 24 hours and NT-proBNP and LVEF at 24 hours and 6 months after AMI. There was a trend towards a positive correlation between VCAN expression and NT-proB- 
Table 3. Statistical estimates for prediction of LVR by VCAN transcript expression

\begin{tabular}{lcccc} 
& AUC $(95 \% \mathrm{CI})$ & P-value & Cut-off & Sensitivity \\
VCAN & $0.67(0.53-0.81)$ & 0.04 & $<75.3 \mathrm{NU}$ & Specificity \\
\hline
\end{tabular}

Abbreviations: AUC, area under the curve; $\mathrm{Cl}$, confidence interval; NU, normalized units; VCAN, versican

Table 4. Results of univariable logistic regression analysis for prediction of LVR including pre-operative VCAN expression transcript above the cut-off value and clinical risk factors

\begin{tabular}{|c|c|c|c|c|}
\hline Variable & OR & \multicolumn{2}{|c|}{$95 \% \mathrm{Cl}$} & $P$-value \\
\hline & & Lower & Upper & \\
\hline VCAN $<75.3$ NU AUC & 4.050 & 1.204 & 13.619 & 0.024 \\
\hline Age & 0.944 & 0.892 & 0.999 & 0.047 \\
\hline Gender (female) & 3.250 & 0.648 & 16.301 & 0.152 \\
\hline BMI & 1.082 & 0.935 & 1.252 & 0.292 \\
\hline Anterior AMI & 2.417 & 0.648 & 9.015 & 0.189 \\
\hline Arterial hypertension & 1.417 & 0.367 & 5.473 & 0.613 \\
\hline Diabetes mellitus & 1.033 & 0.233 & 4.578 & 0.966 \\
\hline Dyslipidemia & 2.885 & 0.556 & 14.957 & 0.207 \\
\hline Smoking & 0.952 & 0.263 & 3.448 & 0.941 \\
\hline Previous AMI & 1.773 & 0.147 & 21.423 & 0.652 \\
\hline NT-proBNP & 1.000 & 0.999 & 1.000 & 0.316 \\
\hline Creatinine & 1.000 & 0.999 & 1.001 & 0.763 \\
\hline Hemoglobin & 1.048 & 0.657 & 1.672 & 0.843 \\
\hline Troponin I & 0.996 & 0.982 & 1.010 & 0.571 \\
\hline LVEDV & 0.992 & 0.972 & 1.012 & 0.421 \\
\hline LVESV & 0.988 & 0.958 & 1.018 & 0.437 \\
\hline LVEF & 0.997 & 0.936 & 1.063 & 0.935 \\
\hline Aspirin & 1.000 & 0.999 & 1.000 & 0.678 \\
\hline P2Y12 inhibitor & 3.621 & 0.726 & 18.066 & 0.117 \\
\hline Statin & 1.000 & 0.999 & 1.000 & 0.520 \\
\hline$\beta$-blocker & 722.712 & 0.001 & 2347.72 & 0.999 \\
\hline ACE-inhibitor or ARB & 686.576 & 0.001 & 6852.21 & 0.999 \\
\hline Aldosterone receptor antagonist & 0.182 & 0.022 & 1.533 & 0.117 \\
\hline
\end{tabular}

Abbreviations: OR, odds ratio; other see Table 2 and 3

Table 5. Multivariable logistic regression model for prediction of LVR including significant predictors of LVR in univariable analysis (pre-operative VCAN expression transcript above the cut-off value and age)

\begin{tabular}{lcccc}
\multicolumn{1}{r}{ Variable } & OR & 95\% Cl & Upper \\
& & Lower & 12.075 \\
VCAN $<75.3$ NU AUC & 3.475 & 1.000 & 0.047 \\
Age & 0.951 & 0.897 & 0.099 \\
\hline
\end{tabular}

Abbreviations: see Table 2-4

NP concentration at 24 hours $(\mathrm{R}=0.23 ; P=0.07)$ and an intermediate, positive correlation at 6 months $(R=0.33$; $P=0.01)$. There were no correlations between VCAN expression and LVEF.

\section{DISCUSSION}

The main finding of our study is that decreased VCAN expression is an independent predictor of LVR after AMI, increasing the odds of LVR 3.5-fold. The second important finding is the positive correlation between VCAN expression and NT-proBNP concentrations in patients after AMI.

VCAN is a protective proteoglycan regulating cell survival and extracellular matrix assembly [11]. Many studies demonstrated the role of VCAN in cardiac remodeling. For example, VCAN was showed to induce myofibroblast differentiation during lung remodeling [14]. In addition, human cardiomyocyte differentiation is accompanied by changes in the expression and accumulation of VCAN [15]. VCAN favors transforming growth factor $\beta$-mediated signaling in cardiomyocytes, thereby accelerating healing of the infarcted myocardium [16]. Upregulation of VCAN in the infarcted myocardium mediates chemotaxis and cytokine release in an animal model. The transient expression of VCAN by infiltrating monocytes in the infarcted area of the heart suggests the role of VCAN in the inflammatory response [17]. Furthermore, a post-mortem analysis of 20 infarcted cardiac muscles demonstrated the similar localization of the fragmented VCAN and ADAM metallopeptidase with thrombospondin type 1 motif 1 (ADAMTS1) in the necrotic areas. ADAMTS1 is produced by infiltrating macrophages, suggesting that inflammatory processes after AMI lead to VCAN fragmentation [18]. Altogether, down-regulation of 
VCAN transcript expression in whole blood samples may be a sign of dysfunctional repair mechanisms after AMI, increasing the risk of LVR.

NT-proBNP is an established biomarker of HF and the fragment of the brain natriuretic hormone (BNP) propeptide, which is released in response to the increased atrial and ventricular wall stretch [19]. BNP secretion counteracts the increased cardiac overload, resulting in vasodilation, diuresis, natriuresis, and inhibition of cardiac remodeling [20]. The positive correlation between VCAN expression and NT-proBNP observed in our cohort indicated that similarly to BNP increased VCAN transcript level might be a protective mechanism in patients after AMI. Concurrently, dysregulated VCAN expression might be a sign of the compensatory mechanisms' failure. However, since we only observed a correlation between both markers, without any causal relationship, we may only speculate about its pathophysiological relevance.

\section{Limitations}

The main limitation of our study is the single-center design and a relatively small sample size included in the analysis. Moreover, since our study was not powered for hard clinical end-points including hospitalizations or death due to $\mathrm{HF}$, our results remain hypothesis-generating and required confirmation in future trials. Finally, the wide confidence interval of VCAN expression in the multivariable logistic regression model requires caution when interpreting the results. Altogether, our results should be confirmed in a larger, preferably multi-center study before implementation in clinical practice.

\section{CONCLUSIONS}

In our cohort of patients after STEMI, low VCAN expression was an independent predictor of LVR at 6 months. Whether decreased expression of VCAN might be a useful tool to predict LVR in clinical practice remains to be established.

\section{Article information}

Acknowledgements: We acknowledge Assist. Prof. Michał Michalak (Department of Computer Science and Statistics, Poznan University of Medical Sciences, Poland) for the statistical consultation.

Funding: National Centre for Research and Development, Poland (grants no. TANGO1/266456/NCBR/2015)

\section{Conflict of interest: None declared.}

Open access: This article is available in open access under Creative Common Attribution-Non-Commercial-No Derivatives 4.0 International (CC BY-NC-ND 4.0) license, allowing to download articles and share them with others as long as they credit the authors and the publisher, but without permission to change them in any way or use them commercially. For commercial use, please contact the journal office at kardiologiapolska@ptkardio.pl.

How to cite: Chabior A, Gąsecka A, Marchel M, et al. Expression of versican mRNA transcript to predict cardiac remodeling after myocardial infarction. Kardiol Pol. 2021 ; 79(7-8): 833-840, doi: 10.33963/KP.a2021.0042.

\section{REFERENCES}

1. Lesyuk W, Kriza C, Kolominsky-Rabas P. Cost-of-illness studies in heart failure: a systematic review 2004-2016. BMC Cardiovasc Disord. 2018; 18(1): 74, doi: 10.1186/s12872-018-0815-3, indexed in Pubmed: 29716540.

2. Fox KF, Cowie MR, Wood DA, et al. Coronary artery disease as the cause of incident heart failure in the population. Eur Heart J. 2001;22(3): 228-236, doi: 10.1053/euhj.2000.2289, indexed in Pubmed: 11161934.

3. Wu QQ, Xiao Y, Yuan Y, et al. Mechanisms contributing to cardiac remodelling. Clin Sci (Lond). 2017; 131(18): 2319-2345, doi: 10.1042/CS20171167, indexed in Pubmed: 28842527.

4. Cokkinos DV, Belogianneas C. Left ventricular remodelling: a problem in search of solutions. Eur Cardiol. 2016; 11(1): 29-35, doi: 10.15420/ecr.2015:9:3, indexed in Pubmed: 30310445.

5. Flachskampf FA, Schmid M, Rost C, et al. Cardiac imaging after myocardial infarction. Eur Heart J. 2011; 32(3): 272-283, doi: 10.1093/eurheartj/ehq446, indexed in Pubmed: 21163851.

6. Orn S, Manhenke C, Anand IS, et al. Effect of left ventricular scar size, location, and transmurality on left ventricular remodeling with healed myocardial infarction. Am J Cardiol. 2007; 99(8): 1109-1114, doi: 10.1016/j. amjcard.2006.11.059, indexed in Pubmed: 17437737.

7. Hallén J, Jensen JK, Fagerland MW, et al. Cardiac troponin I for the prediction of functional recovery and left ventricular remodelling following primary percutaneous coronary intervention for ST-elevation myocardial infarction. Heart. 2010; 96(23): 1892-1897, doi: 10.1136/hrt.2009.190819, indexed in Pubmed: 21062778.

8. Husser O, Monmeneu JV, Sanchis J, et al. Cardiovascular magnetic resonance-derived intramyocardial hemorrhage after STEMI: influence on long-term prognosis, adverse left ventricular remodeling and relationship with microvascular obstruction. Int J Cardiol. 2013; 167(5): 2047-2054, doi: 10.1016/j.ijcard.2012.05.055, indexed in Pubmed: 22682700.

9. Berezin $A E$, Berezin $A A$. Adverse cardiac remodelling after acute myocardial infarction: old and new biomarkers. Dis Markers. 2020; 2020: 1215802, doi: 10.1155/2020/1215802, indexed in Pubmed: 32626540.

10. Cao Y, Li R, Li Y, et al. Identification of transcription factor-gene regulatory network in acute myocardial infarction. Heart Lung Circ. 2017; 26(4): 343-353, doi: 10.1016/j.hlc.2016.06.1209, indexed in Pubmed: 27746059.

11. MaciejakA, Kiliszek M, Michalak M, et al.Gene expression profiling reveals potential prognostic biomarkers associated with the progression of heart failure. Genome Med. 2015; 7(1): 26, doi: 10.1186/s13073-015-0149-z, indexed in Pubmed: 25984239.

12. Ibanez B, James S, Agewall S, et al. ESC Scientific Document Group. 2017 ESC Guidelines for the management of acute myocardial infarction in patients presenting with ST-segment elevation: the task force for the management of acute myocardial infarction in patients presenting with ST-segment elevation of the European Society of Cardiology (ESC). Eur Heart J. 2018; 39(2): 119-177, doi: 10.1093/eurheartj/ehx393, indexed in Pubmed: 28886621.

13. Luchner A, Bröckel U, Muscholl M, et al. Gender-specific differences of cardiac remodeling in subjects with left ventricular dysfunction: a population-based study. Cardiovasc Res. 2002; 53(3): 720-727, doi: 10.1016/s0008-6363(01)00510-7, indexed in Pubmed: 11861042.

14. Carthy JM, Boroomand S, Rahmani M, et al. 130: versican expression induces tissue remodeling. J Heart Lung Transplant. 2009; 28(Suppl 2): S111, doi: 10.1016/j.healun.2008.11.808.

15. Chan CK, Rolle MW, Potter-Perigo S, et al. Differentiation of cardiomyocytes from human embryonic stem cells is accompanied by changes in the extracellular matrix production of versican and hyaluronan. J Cell Biochem. 2010; 111(3): 585-596, doi: 10.1002/jcb.22744, indexed in Pubmed: 20564236.

16. Islam $S$, Chuensirikulchai $K$, Khummuang $S$, et al. Accumulation of versican facilitates wound healing: Implication of its initial ADAMTS-cleavage site. Matrix Biol. 2020; 87: 77-93, doi: 10.1016/j.matbio.2019.10.006, indexed in Pubmed: 31669737.

17. Toeda K, Nakamura K, Hirohata $S$, et al. Versican is induced in infiltrating monocytes in myocardial infarction. Mol Cell Biochem. 2005; 
280(1-2):47-56, doi: 10.1007/s11010-005-8051-4, indexed in Pubmed: 16311904.

18. Pehlivan S, Gurses MS, Ural MN, et al. The role of ADAMTS1 and versican in human myocardial infarction: a postmortem study. Lab Med. 2016; 47(3): 205-212, doi: 10.1093/labmed//mw022, indexed in Pubmed: 27346868.
19. Hall C. NT-ProBNP: the mechanism behind the marker. J Card Fail. 2005; 11(Suppl 5): S81-S83, doi: 10.1016/j.cardfail.2005.04.019, indexed in Pubmed: 15948107.

20. Lee NS, Daniels LB. Current understanding of the compensatory actions of cardiac natriuretic peptides in cardiac failure: a clinical perspective. Card Fail Rev. 2016; 2(1): 14-19, doi: 10.15420/cfr.2016:4:2, indexed in Pubmed: 28848655 